\title{
HIGH RADIO FREQUENCY STUDIES OF \\ EQUATORIAL AND SOUTHERN AGN
}

\author{
M. TORNIKOSKI AND E. VALTAOJA \\ Metsähovi Radio Research Station \\ Metsähovintie 114, FIN-02540 Kylmälä, Finland
}

The Swedish-ESO Submillimetre Telescope (SEST) has been used for the high radio frequency observations of our group's AGN monitoring projects since the end of 1987 .

Our SEST results from October 1987 until June 1994 will be published in $A \mathcal{G} A S$ (in press); the data will be available electronically. The data set consists of 155 sources with the signal-to-noise -ratio of at least one observation (at 90 or $230 \mathrm{GHz}$ ) $\geq 4$.

The sources for which we have data mainly fall into one of the following classes:

- All bright, flat-spectrum quasars between the declination range $0^{\circ}$ to $-25^{\circ}$ (results from our analysis of this sample were published in Tornikoski et al. (1993)).

- All southern blazars (BL Lac objects and high polarization quasars).

- A set of bright, variable, nearly-equatorial sources, which are sources of our long-term monitoring campaign plus several multifrequency campaigns.

- A set of sources from the CGRO-multifrequency collaboration.

These data are being used for studies of various radio properties (spectrum, variability, timescales of variability) of the sources, source classification, Doppler boosting effects, etc.

For the most frequently observed sources we have flux curves which enable us to study the growth and decay of radio outbursts in these sources, the possible differences between the outbursts in one source, and the time delays between high radio frequencies (SEST) and lower radio frequencies (Metsähovi) during the radio outbursts. Examples of two sources with relatively long and well-sampled time series of SEST data, 3C 273 and 1510-089, are shown in Figure 1. 

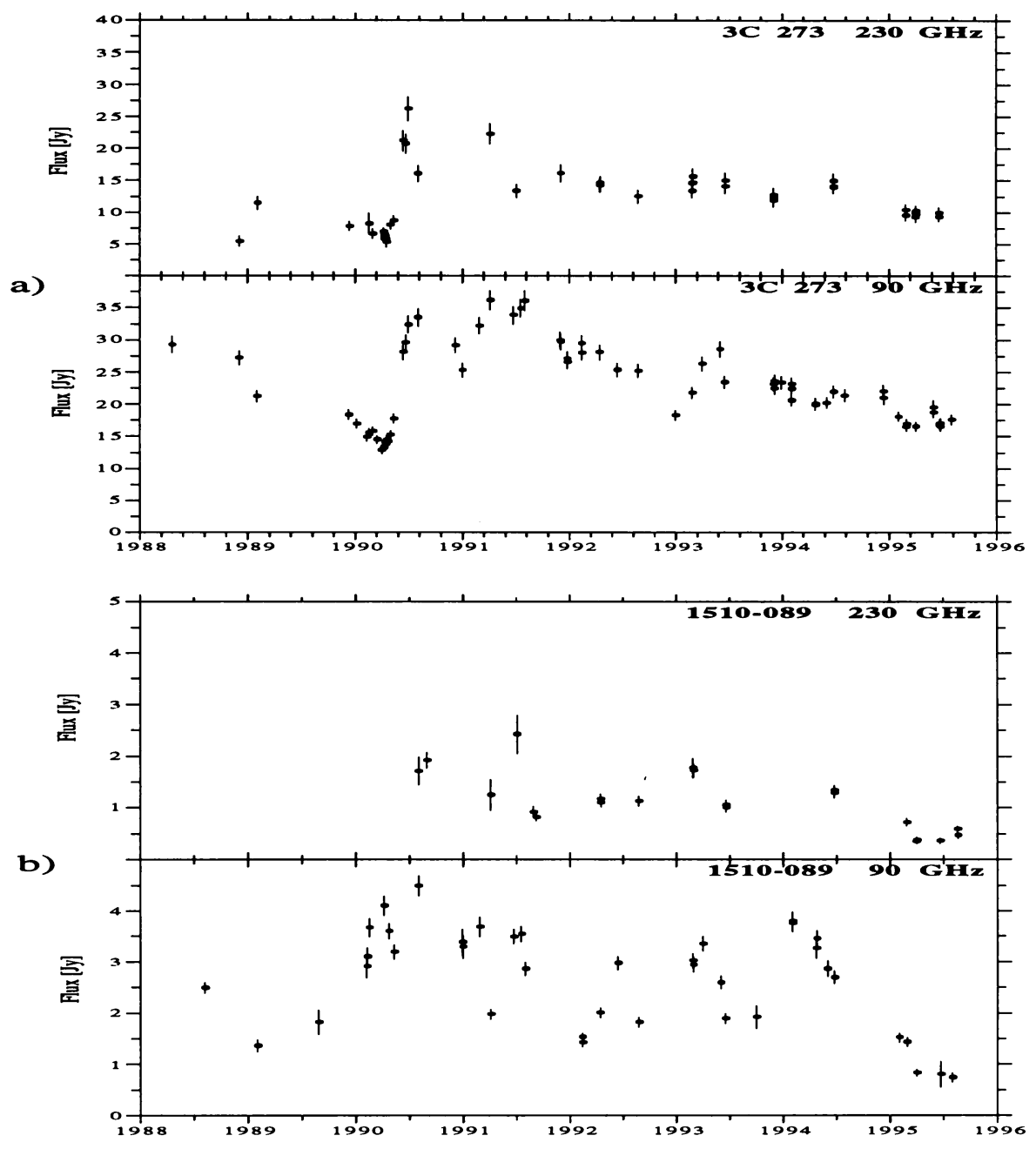

Figure 1. SEST flux density curves of a) 3C 273 and b) 1510-089.

Together with data gathered with other instruments, the SEST data are valuable for understanding the multifrequency behaviour of AGN. The data are used for studies of correlating events at various radiation regimes (cf. Tornikoski et al. (1994), Teräsranta et al. in these proceedings, and several other multifrequency studies).

\section{References}

Tornikoski M., Valtaoja E., Teräsranta H., et al., 1993, $A J, 105,1680$

Tornikoski M., Valtaoja E., Teräsranta H., et al., 1994, $A \& A, 289,673$ 